\title{
e-Migrinter
}

$6 \mid 2010$

Rroms \& Gens du Voyage

\section{Politiques publiques et pratiques d'acteurs : comportements de Voyageurs en réponse à la législation spécifique du stationnement et de la circulation}

\section{Céline Bergeon}

\section{OpenEdition}

\section{Journals}

\section{Édition électronique}

URL : https://journals.openedition.org/e-migrinter/1415

DOI : 10.4000/e-migrinter. 1415

ISSN : 1961-9685

\section{Éditeur}

UMR 7301 - Migrinter

\section{Édition imprimée}

Date de publication : 15 octobre 2010

Pagination : 56-64

ISSN : 1961-9685

\section{Référence électronique}

Céline Bergeon, «Politiques publiques et pratiques d'acteurs : comportements de Voyageurs en réponse à la législation spécifique du stationnement et de la circulation », e-Migrinter [En ligne], 6 2010, mis en ligne le 22 août 2019, consulté le 20 mai 2021. URL : http://journals.openedition.org/emigrinter/1415; DOI : https://doi.org/10.4000/e-migrinter.1415 


\section{Politiques publiques et pratiques d'acteurs : comportements de Voyageurs en réponse à la législation spécifique du stationnement et de la circulation}

Céline Bergeon

\section{$\mathbf{L}$}

a France possède
officiellement depuis 2000 une législation spécifique pour le stationnement des « Gens du Voyage ». Elle oblige les communes de plus de 5000 habitants à créer des aires d'accueil, tandis que les Voyageurs, public visé par la loi, sont astreints à stationner sur ces

lieux identifiés, sous peine de poursuites pénales. Seulement, sept ans après la promulgation de la loi sur « l'accueil et le stationnement des Gens du Voyage ", $32 \%$ des communes ont respecté leurs engagements en termes de lieux de halte.

L'analyse des parcours réalisés par les Voyageurs montrent une organisation réfléchie et stratégique de l'utilisation de

l'espace comme ressource et moyen de reproduction d'un mode de vie. Ainsi, interroger les pratiques circulatoires au prisme des politiques publiques, permet de mettre à jour une circulation « d'avant et d'après législation ». Cette articulation entre mobilité traditionnelle et politiques publiques, redéfinit la circulation et met au jour des comportements circulatoires novateurs.
L'histoire de Manu

En 2004, Manu, manouche sédentarisé de Charente-Maritime, essaie lors d'un entretien de me décrire précisément ce que représente pour lui «la vie de bohème », expression qu'il vient d'employer pour caractériser le voyage. Il m'explique non sans difficulté que le voyage "c'est eux», "que c'est la liberté», qu'en tant que gens du voyage, ils «voyagent» et qu'ils se définissent ainsi par rapport aux autres, les sédentaires, ceux qu'ils appellent les "Gadjé». Le discours de Manu, presque mystique, place la pratique du voyage comme élément fondateur d'un mode de vie qui participe d'un bien être physique et psychologique: d'une part la circulation permet d'instaurer un équilibre au sein de la vie communautaire; mais d'autre part, elle est source de différenciation (par rapport à la société globale) et d'identification au groupe d'appartenance. L'histoire personnelle de Manu illustre tout à fait l'emboitement entre des pratiques culturelles liées au voyage et exprimées dans un cadre communautaire et des relations conflictuelles mais parfois nécessaires avec les «Gadjé ». Pendant sa jeunesse, Manu a circulé avec ses parents et ses grands-parents dans l'Est de la France. C'est une famille dont la mobilité s'exprime à l'échelle régionale (ceux que certains appellent les «Voyageurs régionaux»). Pendant la Seconde Guerre mondiale, Manu et ses parents fuient l'Est de la France et viennent s'installer en Bretagne. Ce départ vers l'Ouest, relevant d'une migration plus ou moins forcée, oblige Manu et ses proches à reconstituer un potentiel circulatoire local. En effet, ils arrivent dans un nouvel espace dont leurs connaissances en termes de potentialités économiques sont restreintes. 
C'est au bout de quelques mois que leur circulation commence à s'organiser après l'identification des ressources locales à exploiter mais également après la détermination d'une certaine demande locale en termes de services auxquels ils peuvent répondre. Le pouvoir d'adaptation de la famille de Manu est donc important. Très vite, les besoins locaux sont identifiés et les réseaux économiques sont crées. Les logiques de circulation sont les suivantes : la côté bretonne est investie pendant la période estivale, l'intérieur des terres pendant l'hiver et les saisons agricoles :

\section{L'été on était sur le littoral et l'biver à lintérieur des terres. Par contre, il faut circuler économiquement, c'est qu'il ne faut pas qu'il y ait trop de Voyageurs au même endroit, on fait presque quand même tous les mêmes activités, les demandes ca court pas non plus, si il y a trop de concurrence et trop d'offre, y aura forcément des Voyageurs qui gagneront moins que les autres. Dans ces cas- là il faut partir. On était rendu à un point où on devait faire des métiers spéciaux pour se démarquer car à peu près tout le monde faisait les mêmes activités. (Terrain 2004,} Charente Maritime, J.J, homme Manouche en reprise du Voyage)

Dans la famille, la confection et la vente de paniers en osier est une activité qui se transmet depuis plusieurs générations. La qualité du produit, le savoir-faire font la fierté de la famille. Quelques années plus $\operatorname{tard}{ }^{1}$, l'ensemble de la famille décide de quitter la Bretagne et de rejoindre le centreouest de la France, des cousins issus d'une autre famille du groupe y résident. Un nouvel espace de vie se forme alors entre la

\footnotetext{
${ }^{1}$ La conception et la référence au temps chez les gens $\mathrm{du}$ voyage est particulière. Leurs repères ne s'articulent pas autour de dates mais se réfèrent à des événements ponctuels comme «l'année où la ferraille a bien marché » ou bien encore «l'année où la Lisa est né ». Je ne m'attarderai pas ici sur ce sujet puisque ce n'est pas le thème traité mais le lecteur pourra se rendre compte des difficultés que peut occasionner une telle conception du temps pour un travail de terrain de qualité, à tout le moins précis.
}

région Poitou-Charentes et le Limousin. De nouvelles alliances économiques sont crées. À ses 18 ans, Manu effectue comme tout citoyen français, son service militaire. Ce moment est une période charnière dans sa vie puisqu'après avoir "goûté » au confort de la sédentarité, Manu décide à cette époque de se sédentariser d'autant plus que le reste de sa famille a lourdement amoindrie sa circulation. Le décès de son épouse le conforte encore plus dans sa décision de se fixer. Il devient donc un Voyageur sédentaire, qui au fil des années, emprunte de plus en plus d'habitudes individuelles liées à un mode de vie sédentaire. Néanmoins, Manu garde toujours ce sentiment inexplicable de l'attachement au voyage. Bien plus tard, il rencontre Louise, une Gadgi, résidant en Charente-Maritime. Professionnellement, il est engagé dans une association travaillant auprès de la population des gens du voyage. Il s'occupe de l'atelier vannerie de l'association. Il peut ainsi vivre de son savoir faire au sein d'une structure professionnelle dite «normale ». Manu précise que, bien qu'il soit sédentaire, son quotidien est entouré d'emprunts au mode de vie nomade. Il travaille son savoir faire, les caravanes et les Voyageurs l'entourent quotidiennement, il parle le manouche. À la veille de sa retraite, il nous informe qu'après discussion avec sa femme, ils décident de reprendre la route, de se reconstruire un capital circulatoire. Il s'achète une petite caravane, puis une plus grande, à deux essieux, il s'équipe d'un camion. Il fréquente de plus en plus son cousin sédentarisé en Dordogne, qui souhaite également reprendre le voyage. Les longues discussions sur une éventuelle reprise alimentent un souhait de plus en plus fort. Il me montre sa carte permettant l'exercice d'activités non sédentaires. Il garde sa maison pour les avantages liés à la sédentarité notamment au niveau des procédures de contrôles. Il est manouche, également français mais Voyageur avant tout.

L'histoire de Manu, illustre de façon pertinente que la circulation décrit bien plus 
qu'une pratique, elle fédère un mode de vie et un état social. L'arrêt de la mobilité ne réinterroge pas les sentiments d'appartenance et d'attache au groupe «Voyageurs». De par les discours des acteurs, elle s'érige en équilibre social.

\section{Une circulation légiférée, un mode de vie attaqué}

Un paradoxe existe dans les sociétés sédentaires qui sont amenées à gérer des populations dont la mobilité est le mode de vie. D'un côté, la circulation d'une partie de la population, qui en a fait son quotidien, dérange; d'un autre côté, le contexte économique actuel, en parallèle au développement des sociétés, appellent les citoyens à élargir leur potentiel mobile. Bien que cette mobilité contemporaine ne puisse s’apparenter à celle des premiers (Knafou, 1998: 12), elle témoigne de l'évolution des pratiques circulatoires, tant dans leurs formes que dans leur nombre. Si le mouvement est aujourd'hui considéré comme une capacité d'adaptation, celui-ci est remis en cause quand il organise un mode de vie. C'est le cas de la population des gens du voyage. Je prendrai l'exemple de la France dans sa manière d'élaborer des lois, mises en application par la suite à des échelles d'intervention plus grandes: je l'illustrerai par un travail de terrain effectué dans la région Poitou-Charentes dans le cadre de ma thèse $^{2}$. Les comportements et les pratiques des gens du voyage développés en réponse à ces recommandations, feront l'objet d'une attention particulière.

Le contexte répressif envers les populations nomades s'installe véritablement par des mesures concrètes pendant la seconde moitié du 19ème siècle. Mais c'est

\footnotetext{
2 Céline Bergeon: Rroms et Voyageurs: pratiques circulatoires et attaches territoriales an miroir des politiques publiques de stationnement et de circulation: l'exemple des Rroms du Poitou-Charentes (France) et de la région Wallonne (Belgique). Thèse de Doctorat en cours.
}

au cours du siècle suivant, que les textes législatifs se succèdent et instaurent le nomade dans un statut et un cadre juridique spécifique (Robert, 2007: 122). Ces textes concernent principalement les modalités selon lesquelles la circulation doit être pratiquée et les conditions d'exercice des activités économiques ambulantes, connexes au mode de vie circulant. La loi du 16 juillet 1912 instaure le "carnet anthropométrique », document qui devait être possédé par chaque personne en habitat mobile et ne justifiant d'aucun revenu fixe. Un visa à chaque départ et à chaque arrivée dans les communes devaient y être apposé par les forces de l'ordre. A cela s'ajoute une demande d'autorisation journalière pour stationner dans la commune Chaque jour, les familles nomades doivent obtenir l'autorisation de stationner pendant vingt-quatre heures en un lieu désigné par les autorités communales (Humeau, 1995: 65). Une période «d'itinérance surveillée » débute. La mobilité, appréhendée comme de l'errance à cette période, était considérée comme un manque, à tout le moins, comme une pratique dangereuse. Dès lors, la société d'accueil les a qualifié de populations indésirables notamment par l'illégalité de leur mobilité, associée au vagabondage (Blum Le Coat; Catarino; Quiminal, 2004: 159). La loi de 1912 est abrogée suite à la mise en application de celle du 03 janvier 1969. Les carnets anthropométriques sont supprimés, cependant, les Voyageurs doivent avoir en leur possession un titre de circulation. Il en existe trois versions différentes : le livret de circulation, attribué aux sans domicile fixe depuis plus de six mois, qui vivent de façon permanente dans une habitation mobile et qui disposent de ressources régulières; le livret spécial de circulation octroyé aux sans domicile fixe depuis plus de six mois, qui exercent une activité professionnelle ambulante avec une inscription au registre du commerce; et enfin le carnet de circulation qui est attribué aux sans domicile fixe qui vivent de façon permanente dans une habitation mobile et qui ne justifient d'aucune ressource 
financière fixe. L'encadrement de la circulation se fait par conséquent par l'attribution d'un titre de transport, lequel doit être visé tous les trois mois par une instance aux pouvoirs délégués telle que la gendarmerie. Le statut actuel des gens du voyage en France est donc cadré par la loi du 03 janvier 1969 qui les oblige à posséder un document officiel attestant de leur «nonsédentarité ». L'instauration de ces titres de circulation a été une mesure spécifique mise en place pour contrôler au mieux cette population au sein de l'espace national. Elle reste discriminatoire malgré l'abandon du carnet anthropométrique (Robert, 2007: 125). Ces documents sous-entendent également un contrôle plus systématique des ressources financières des Voyageurs. En parallèle à ce dispositif, toute demande de titre de circulation doit être accompagnée d'une demande de rattachement communal, qui décrit de façon insidieuse une "pseudosédentarité » où le législateur crée de l'inscription territoriale officielle afin de pallier la nomadité des groupes de gens du voyage. A chaque fois, la problématique est la même: il s'agit de limiter leurs déplacements pour mieux les contrôler, de nier les réalités de la vie nomade, et de tenter de la faire "coller» avec les fonctionnements de la vie sédentaire (Carrère, Daadouch, 2000).

Enfin, la loi de 2000, dite loi Besson II instaure des aires officielles de stationnement. Les communes de plus de 5000 habitants doivent se doter d'espaces de stationnement conformes pour l'accueil des populations circulantes. Dans un même temps, les Voyageurs sont dans l'obligation de s'arrêter dans ces lieux sous peine d'expulsion et de poursuites. La création d'un dispositif d'accueil procure ainsi aux maires des communes un pouvoir de refoulement. Au 31 décembre 2007, soit sept ans après promulgation de la loi, seulement $32 \%$ des espaces de stationnement, prévus par l'ensemble des schémas départementaux est réalisé (Hérisson, 2008). Obligation de stationnement et pénurie d'espaces d'accueil rythment donc le quotidien des familles de gens du voyage en France qui doivent composer entre une circulation balisée, imposée par la société et une circulation pensée et organisée en termes de mode de vie. Les enjeux sont opposés : le législateur souhaite contrôler une minorité dont la mobilité est considérée comme suspicieuse. A l'opposé, les Voyageurs désirent perpétuer leur circulation, véritable équilibre socioéconomique et psychologique. En effet, le groupe et le voyage sont deux éléments particulièrement liés et offrent une certaine sécurité à ceux qui en font partis (Formoso, 1987 : 192). Bien plus qu'un mode de vie, c'est l'ensemble des savoirs faire qui sont aujourd'hui soumis à l'évolution. Le « savoir circuler» devient orienté, le savoir faire professionnel lié à l'itinérance est fondamentalement modifié, la transmission inter-générationnelle est réinterrogée et implicitement la reproduction de la circulation est remise en cause.

\section{Quelles répercussions sur la circulation?}

Il serait aisé de considérer que les espaces de stationnement sont crées dans le but d'offrir des conditions décentes aux Voyageurs pour leur arrêt. C'est pourtant un des objectifs premiers de la loi Besson. Néanmoins, sa non-application (32\% des aires d'accueil réalisé en 2007), sous-entend que le désintérêt relève d'une autre problématique. Le manque d'espaces de stationnement engendre des problèmes puisque les Voyageurs sont obligés de s'arrêter sur des espaces qui n'existent pas. Dans un même temps, lorsque ces lieux existent, leur localisation ne correspond pas forcément aux parcours des groupes. C'est ainsi que certaines aires d'accueil sont surfréquentées et d'autres délaissées. Le seuil des 5000 habitants pour la création de lieux de halte relève d'un choix arbitraire de la part des institutions. Cependant, l'obligation de stationnement sur des espaces identifiés pousse certaines familles à désinvestir les lieux initiaux du parcours dans lesquels les réseaux économiques et sociaux sont déjà établis. Par ailleurs, une présence trop 
importante de Voyageurs sur un même espace, engendre de la «concurrence négative » dans le sens où l'offre de main d'œuvre (qui est ici ponctuelle dans le cadre du nomadisme) reste beaucoup plus élevée que la demande, émanant de la population locale, en termes de services. Les aires d'accueil ont donc de réelles répercussions sur les schémas d'itinérance puisque qu'elles dé-territorialisent une population déjà inscrite spatialement par leurs habitudes de circulation et par leurs réseaux socioéconomiques. Le voyage est un des éléments fondateurs d'un mode de vie et lorsqu'il est remis en cause, c'est l'ensemble des pratiques adhérentes qui sont bouleversées. La législation du stationnement se répercute également sur les habitudes quotidiennes. En parallèle à la création des espaces d'accueil, de nombreuses recommandations concernant la santé ou encore la scolarisation sont mentionnées. Comme si, résoudre «le problème » de la circulation pouvait normaliser l'ensemble des pratiques quotidiennes liées au voyage. Les conséquences des politiques publiques suivent un schéma identifiable. Le législateur est en quête d'une normalisation d'un mode de vie.

Une législation est spécifiquement créée pour maîtriser et réguler la circulation, pratique fondamentale d'un groupe minoritaire. Étant donné que l'ensemble des activités connexes s'articulent autour du mouvement, c'est ce dernier lui-même que les pouvoirs publics voudraient faire évoluer.

Plusieurs logiques se mettent en place. D'un côté celui de la territorialisation forcée, obligeant les familles et les Voyageurs à fréquenter des lieux jamais ou presque jamais investis; d'un autre côté, le manque d'espaces de stationnement les positionne dans une situation continuelle de quête d'un lieu de halte: le caractère organisé de la circulation que peut conférer le mode de vie circulant peut se transformer en errance. J'ajouterai ici, que cette errance n'est pas seulement géographique, elle est aussi errance sociale et économique puisque les réseaux sont désorganisés : l'espace tsigane [...] c'est aussi et surtout, un lieu de vie, ouvert sur des pratiques sociales, économiques, relationnelles, maîtrisées par ceux qui les déploient. Ce lieu, c'est l'endroit où les membres d'une communauté ont leurs habitudes, là où ils ont tendance à se retrouver (Reyniers, 2005: 70). Concrètement, investir un nouveau lieu nécessite la création de liens sociaux nouveaux afin de pouvoir poursuivre des activités économiques traditionnelles. Celles-ci sont principalement axées sur l'échange avec les Gadjé et même si les Voyageurs semblent répondre à une demande ponctuelle de services et de produits commerciaux, la fidélisation de la clientèle est récurrente.

L'a-territorialité supposée, due à la circulation régulière, incite les institutions à territorialiser ces populations, considérées comme sans attache, par la création de lois qui instaurent elles-mêmes de la fixité. En effet, la création de points de fixité instaure d'une part de la fréquentation balisée et d'autre part des difficultés réelles à la reproduction des schémas classiques d'itinérance. Pour de nombreux Voyageurs, les aires d'accueil ont introduit une gêne dans leur circulation au point que certains ne puissent plus voyager. Cette "gêne» mentionnée par les familles relève du fait que l'ensemble des législations ont pour vocation implicite leur sédentarisation et leur assignation à résidence. Paradoxe de la législation, les aires d'accueil ont été créées pour mieux contrôler les Voyageurs et de fait les inviter à la sédentarité. Pourtant, les règlements intérieurs des aires d'accueil sont stricts: les durées de stationnement ne peuvent excéder quelques mois. C'est ce que précise la circulaire relative à l'application de la loi Besson de 2000: La durée maximum de séjour autorisée est définie an vu de l'évaluation des besoins du schéma départemental. Elle est précisée dans le règlement intérieur de l'aire d'accueil. Elle doit être adaptée aux capacités d'accueil sur la commune ou les communes environnantes, prévues par le schéma. D'une manière générale, le règlement intérieur ne doit pas prévoir une durée continue de 
séjour supérieure à neuf mois, ce qui n'exclut pas la possibilité de dérogation en cas de situation particulière (hospitalisation de longue durée d'un membre de la famille, activité professionnelle par exemple) (Source : Circulaire UHC/IUH1/12 no 2001-49 du 5 juillet 2001 relative à l'application de la loi no 2000-614 du 5 juillet 2000 relative à l'accueil et à l'habitat des gens du voyage).

\section{De la résilience au développement de comportements dynamiques : l'autonomie dans la circulation}

La mise en place des lois sur le stationnement et la circulation ${ }^{3}$ des Voyageurs ont de réelles répercussions sur les pratiques des groupes. Le travail de terrain effectué en Poitou-Charentes et en région wallonne a permis de voir la diversité des comportements développés en réponse à ces politiques publiques.

Premièrement, certaines familles s'inscrivent dans une logique de «soumission» et modifient leur mobilité. Les pratiques circulatoires deviennent orientées spatialement. C'est l'objectif implicite et premier du législateur avec la mise en place des lois sur le stationnement. La halte devient compliquée et «brouille» le potentiel mobile des groupes. La sédentarisation est alors considérée comme la seule issue possible. Dans ce cas précis, l'arrêt de la mobilité n'est pas uniquement le fruit d'une précarisation des familles, ce sont les conditions et les modalités dans lesquelles la circulation peut être pratiquée qui la rendent complexe. Les principales

\footnotetext{
3 La loi du 3 janvier 1969 sur l'exercice des professions ambulantes vient également rendre complexe la poursuite de la circulation. Néanmoins, le problème des aires d'accueil, lieux de halte artificielle paraissent être le souci majeur pour les familles de Gens du Voyage encore circulantes. Les difficultés économiques viennent renforcer cette gêne. La polyvalence, l'adaptabilité professionnelle et économique qui leur sont reconnues (Reyniers, 2005 : 66) nous permettent d'avancer que les problèmes liées aux conditions de haltes précédent les difficultés économiques.
}

activités économiques des familles étant dépendantes de la mobilité, la sédentarité implique leur restructuration. L'équilibre que peut conférer le voyage aux activités économiques est rompu, les savoir-faire doivent être pensés autrement. Les familles doivent s'organiser à présent selon la fixité. L'impact de la sédentarisation sur l'unité et la cohésion du groupe est réel. Différents comportements peuvent se développer suite à l'arrêt de la circulation. Celui du repli communautaire où toutes relations, pourtant utiles, avec la société environnante sont rompues: c'est le cas par exemple d'un groupe de manouche de Charente-Maritime, qui en 2004, s'appropria un espace de stationnement géré par l'APTGV ${ }^{4}$. Les médiateurs sociaux de ce centre social, ne pouvaient plus s'y rendre, le climat était devenu dangereux. Cet espace de stationnement était devenu une zone de non-droit. $\mathrm{Ou}$ encore celui de l'individualisation des comportements. En effet, certains groupes qui décident d'arrêter le voyage, acquièrent au fur et à mesure du temps, des habitudes individuelles. Le souhait de se retrouver en couple sur un terrain, ou celui que les enfants prennent leur indépendance est parfois une demande de certains Voyageurs. C'est le cas de ce couple résident en Dordogne: après une sédentarisation récente et plus ou moins voulue, ce groupe est composé de la «Manmie » (Grand-Mère), de ses enfants qui eux-mêmes ont eu des enfants. Ils résidaient tous sur un même terrain familial en 2005. C'est lors d'une rencontre en CharenteMaritime, à l'occasion d'une réunion familial pendant l'été, que la fille de la Manmie s'est livrée. Elle a confié qu'elle avait de plus en plus de difficultés à vivre à proximité directe avec ses enfants, qu'elle souhaiterait maintenant que ses enfants aient leur propre terrain afin d'être un peu plus tranquille. Alors, avec son mari, elle a décidé de vendre le terrain sur lequel ils résidaient tous ensemble pour faire l'acquisition d'une autre

4 APTGV: Association pour la Promotion des Tsiganes et des Gens du Voyage - Mise en liquidation judiciaire en 2007 par le Tribunal de Saintes (17) 
propriété plus petite où ils pourront vivre avec plus de tranquillité. Cette individualisation des comportements montrent combien l'abandon de la circulation met les groupes dans une "conformité inconsciente». Cependant, pour la plupart, la sédentarisation implique une redéfinition du quotidien mais ils restent néanmoins très attachés et ancrés dans des pratiques liées au voyage. Par ailleurs, bien que des logiques d'individualisation des comportements aient été reportées avec le travail de terrain, la plupart des groupes rencontrés maintiennent des relations familiales soutenues.

Deuxièmement, certains groupes s'inscrivent dans une logique de refus. Ils contestent le stationnement balisé instauré par le législateur et défendent par le stationnement illicite leur mode de vie et leur droit d'aller et venir. Bien que la pratique de l'arrêt illicite prenne sa source dans la pénurie d'aires d'accueil, certaines familles refusent toute implantation identifiée; d'une part parce qu'elles choisissent de privilégier l'équilibre de leur circulation, d'autre part parce que les conditions de stationnement sur des espaces d'accueil ne leur conviennent pas: la cohabitation inter-groupe dans ces lieux est fréquemment problématique et certains y refusent de s'arrêter pour ces raisons. Le refus est alors considéré comme contestation publique.

Enfin, et c'est dans ce contexte que l'adaptabilité qui est souvent associée aux Voyageurs s'exprime à mon avis de façon pertinente, des groupes s'inscrivent dans une logique dynamique en tant que créateurs d'initiatives face aux gênes occasionnées par les politiques publiques de stationnement. Ces initiatives peuvent prendre plusieurs facettes. Dans un premier temps, les groupes inscrivent leur savoir-faire professionnel issus de la circulation dans la fixité. Des Voyageurs décident ainsi de créer des microentreprises dans lesquelles ils proposent leur savoir-faire et des produits issus d'un mode de vie circulatoire. Ils peuvent ainsi exercer de façon légale des activités liées à leur commerce ou à leur artisanat traditionnel. Ce type d'entreprise implique plus ou moins de sédentarité selon la nature du produit et/ou du service proposé. Certains Voyageurs possèdent une micro-entreprise de vannerie par exemple et continuent d'être sur les routes.

Dans un second temps, la gêne occasionne des initiatives circulatoires. Certains groupes vont modifier leurs parcours et créer des stratégies spatiales pour pérenniser un mode de vie. Ils composent avec la législation en se la réappropriant, en la contournant de manière à s'inscrire dans une logique circulatoire, une "mobilité adaptée ». C'est le cas de Manu, qui a gardé son domicile et qui vend des paniers sur les marchés, selon un calendrier identifié. Les retours au domicile peuvent se faire de façon quotidienne (s'apparentant à des mouvements pendulaires) ou de manière plus ponctuelle. Manu part souvent pendant la période estivale et ne revient chez lui qu'un ou deux mois plus tard. Les voyages d'une semaine sont également fréquents.

Cette reformulation de la mobilité ne permet pas aux Voyageurs de reproduire un mode de vie basé exclusivement sur le nomadisme et l'itinérance, elle décrit une évolution importante des pratiques liées à la circulation et c'est dans un contexte légiféré qu'ils sont aujourd'hui amenés à circuler. Leur motilité en tant que capacité à se déplacer est réduite par l'instauration d'un stationnement balisé. En effet, leurs potentialités mobiles sont amoindries si on considère que la capacité à se mouvoir est le facteur clé définissant la mobilité (Kaufmann, 2007: 179-180). À cela s'ajoute la complexification des procédures liées à l'exercice des activités professionnelles ambulantes qui pose d'importantes difficultés aux Voyageurs. Le choix et l'autonomie des familles sont donc essentiels dans la reproduction des pratiques circulatoires. 


\section{Le choix et l'autonomie dans les pratiques : deux facteurs essentiels}

L'analyse des répercussions des politiques publiques de stationnement et de circulation sur la mobilité des Voyageurs met en lumière deux critères importants: le choix et l'autonomie dans les pratiques circulatoires.

La notion de choix est importante tant dans le processus de sédentarisation que dans l'acte de la circulation. Le travail de terrain m'a permis de mettre en lumière la manière dont est vécue la sédentarité quand celle-ci relève d'un choix volontaire. En effet, quand elle est voulue, la halte prolongée est vécue de façon plus sereine contrairement aux familles qui s'arrêtent pour des difficultés inhérentes à leur mode de vie. Lorsqu'elle relève d'un choix, la sédentarisation est considérée comme une période dont la durée n'est pas forcément indéterminée. En effet, le voyage reste présent dans la vie quotidienne (habitat caravane, ou bien encore organisation des relations sociales journalières) et semble pouvoir être repris à tout moment si la décision en est prise. A l'opposé, lorsque la sédentarisation découle d'une impossibilité de continuer la circulation du fait de problèmes économiques par exemple, l'arrêt définitif reste mal vécu. La sédentarisation est vue comme une soumission sociale où les repères culturels s'amoindrissent, voire disparaissent.

Par ailleurs, la question du choix est essentielle dans la logique propre du parcours. Comme je l'ai précisé avec la circulation balisée introduite par les aires d'accueil, des espaces «non apprivoisés» socialement et économiquement sont investis par des familles. Les réseaux socioéconomiques qu'elles ont l'habitude d'utiliser lors de leurs venues régulières sont absents. Hors, je l'ai précisé à plusieurs reprises, ce sont précisément ces réseaux qui permettent l'échange économique et participent de la pérennité économique du parcours.
L'autonomie des groupes se comprend dans le sens où l'entend Emmanuel Ma Mung dans son Habilitation à diriger des Recherches: le point de vue de l'autonomie appliqué à l'étude de populations localisées, c'est-àdire, situées dans l'espace, consisterait donc à voir comment les groupes bumains se constituent et se maintiennent dans leurs relations avec ce qu'ils ne sont pas (autres groupes, autres espaces); comment ils se définissent dans les relations avec ce qui leur apparait, et qu'ils se donnent, comme leur altérité sociale et spatiale (Ma Mung, 1999: 13 ). La dynamique introduite par le processus de différenciation - entre les Voyageurs et le Gadje $^{5}$ - participe de la reproduction culturelle des sociétés. L'autonomie se retrouve dans les projets de circulation et dans les objectifs économiques. Être autonome n'engendre pas l'exclusion, j'entends ici non pas le fait que certains groupes vivent indépendamment de ce qui les entoure puisque le pouvoir dominant a forcément une emprise sur les groupes minoritaires par les lois qu'il impose. Je le comprends dans le sens où c'est le groupe qui décide de son inscription sociale et spatiale, il délimite son espace relationnel et les frontières de cet espace. La préservation et la transmission de la différence s'illustrent dans le quotidien par la langue, les pratiques spatiales, l'habitat... mais surtout par la mise à distance de ceux qui n'appartiennent pas à la communauté, "les Gadjé ». L'identité du groupe se déploie à travers l'espace pratiqué. Le nomadisme décrit un rapport à l'espace fréquent car il repose sur de la mobilité. Circuler, «nomadiser » revient à reproduire son identité.

Les politiques publiques de stationnement et de circulation participent de l'amoindrissement des possibilités liées au choix et à l'autonomie des familles dans l'acte circulatoire. L'idée principale de cet article repose sur une volonté d'exposer les dynamiques engendrées par de la gêne

\footnotetext{
${ }^{5}$ Il existe aussi des processus de différenciation entre les groupes de gens du voyage selon le degré de mobilité, l'origine supposée et selon les activités économiques.
} 
politique. Certes, les conséquences sont importantes en termes de reproduction traditionnelle des pratiques. Néanmoins, elles introduisent de la réaction par l'adaptation et l'initiative. Elles provoquent un enjeu d'affirmation des identités minoritaires au sein des espaces nationaux.

Céline Bergeon

Doctorante en géographie

Migrinter - UMR 6588

CNRS /Université de Poitiers celine.bergeon@,univ-poitiers.fr

\section{Bibliographie}

Blum Le Coat, Jean-Yves ; Catarino, Christine ; Quiminal Catherine (2004) Les Gens du Voyage : errance et prégnance des catégories, in Gotman, Anne; Assier-Andrieu, Louis (dir.), Villes et Hospitalités: les municipalités et les étrangers, Paris, Ed. de la Maison des Sciences de l'Homme, pp. 158-176.

Carrère, Violaine ; Daadouch Christophe (2000) Les Gens du Voyage en mobilité surveillée, Plein Droit, n 46 , septembre 2000, pp. 5-10.

Formoso, Bernard (1987) Tsiganes et sédentaires : la reproduction sociale d'une société, Paris, l'Harmattan, 262 p. (Connaissances des Hommes).

Humeau, Jean-Baptiste (1995) Tsiganes en France, Paris, l'Harmattan, 409 p.

Kaufmann, Vincent (2007) La motilité, une notion clé pour revisiter l'urbain? in Bassand, Michel ; Kaufmann, Vincent; Joye, Dominique (dir.), Enjeux de la sociologie urbaine, Lausanne, Presses polytechniques et universitaires romandes, pp. 1717-188.

Knafou, Rémy (1998) La planète "nomade ». Les mobilités géographiques d'aujourd'bui, Paris, Belin, 247 p. (Festival international de géographie de Saint-Dié-des-Vosges).

Ma Mung, Emmanuel (1999) Autonomie, migrations et altérité, Poitiers, Université de Poitiers, 448 p.

HDR : Géogr. : Poitiers : 1999.

Reyniers, Alain (2005) Migrations, mobilité et territorialité chez les tsiganes, in Dobrenko, Bernard (dir.), Territoires et minorités : la situation des gens du voyage, Limoges, Pulim, pp. 63-72.

Robert, Christophe (2007) Eternels étrangers de l'intérieur, Paris, Desclée de Brouwer, 452 p. 